\title{
Sway: An Adaptive Travel Platform
}

\author{
Isabel Dec \\ IIT Institute of Design \\ Chicago, IL 60661, USA \\ idec@id.iit.edu
}

\author{
Evan Chan \\ IIT Institute of Design \\ Chicago, IL 60661, USA \\ echan9@id.iit.edu
}

\author{
Santosh Basapur \\ IIT Institute of Design \\ Chicago, IL 60661, USA \\ basapur@id.iit.edu
}

\begin{abstract}
With an overwhelming amount of information, transportation is one of the most complex challenges we tackle each day. You need to be somewhere; how will you get there? What mode of transportation will you take? Which route? Check the weather. Check the traffic. Check the bus/train schedule. Check the Uber/Lyft prices. The list goes on and on. The task of trip planning has become quite cumbersome because each person has their own unique set of preferences. These preferences determine which modes of transport and which routes are preferred over others in any given circumstance. This paper shares our findings as we attempted to gain an overarching understanding of travel preferences and presents our concept for a new platform that accounts for them. Through personalization, the interface presents users with the information they would want to know and hides everything else that's irrelevant to them.
\end{abstract}

Urban Mobility, Complex Systems, Transportation Futures, $\mathrm{HCl}$, Interaction Design, iUl, Adaptive Systems.

\section{INTRODUCTION}

Transportation is fraught with complexity. Most areas are an intricate lattice of roads that provide numerous alternatives for getting from point $A$ to point $B$; which one is the most appropriate? This quandary is amplified in metropolitan areas where the list of viable modes of transportation expands with the addition of public (e.g. bus, subway) and private (e.g. Uber/Lyft) options. The introduction of more transportation alternatives has led to a corresponding increase in travelers' willingness to utilize multiple modes of transportation [6,9]. In most U.S. cities, trip planning, which is the decision-making that goes on before a traveler decides what transportation modes and routes to utilize, is largely done on smartphones at present [5]. Google Maps [8] and Citymapper [2] are two of the most frequently used mobile applications for trip planning. Both attempt to provide accurate and easily-accessible information for all modes of transportation.

\section{LITERATURE REVIEW}

Most of the work in this area is focused on three things 1) route optimization $[7,10] 2)$ sharing economy [6] and 3) highly specialized transportation needs like that of seniors or environment conscious travelers [10, 11]. Mobile applications for route optimization, such as Google Maps and Citymapper, use input of two locations and display the route(s) for each mode of transportation with the shortest amount of travel time. Our observation from user interviews analyzing these two apps suggested that time/ duration of travel isn't the only criterion that is considered in the decision-making process. There are many additional variables people prefer by which to optimize their travel.

The Intelligent Transportation Systems (ITS) community has worked on route optimization on an algorithmic level [3,4]. Dibbelt et al's. eCompass [3] detailed algorithms that enable 1) multimodal public transportation route planning and 2) personalized route optimization for tourists with accommodations for lunch, coffee breaks, and such. Digmayer et al. [4] surveyed the ITS literature and summarized that travel information applications should support user activity before, during, and after trips using transportation systems. They emphasized the use of scenario-based activity analysis for supporting people.

The $\mathrm{HCl}$ community has addressed issues like optimizing transportation for senior citizens [11], optimizing transportation for $\mathrm{CO}_{2}$ emissions [10], and shared economy and its influences [6]. Unfortunately, to date we see a lack of applications for universal use that optimize for disparate individual differences in transportation choices. Additionally, there seems to be a shortage in experimentation with new design concepts for such complex applications. 


\section{RESEARCH METHODOLOGY}

Research questions were framed through literature review and team's interest in metropolitan transportation and interaction design:

R1: How do city dwellers/commuters utilize current mobile travel applications? What are their attitudes toward the features they provide or not?

R2: What are commuters' criteria for choosing a certain mode(s) of transportation over others? Or how/why do they use a combination of modes when available?

\subsection{Methodology and Initial Findings}

We started by observing transportation hubs for activities done by travellers-3 bus stations, 3 subway stations, and 3 Divvy bike-share stations. Then we interviewed 15 people of various ages (ages 20-60) to get insight into their trip planning; what is the decision-making that goes into choices of modes of transport and routes taken. We covered both city and surrounding suburban areas (see Figure 1 for full list) and used the friend-of-afriend approach to recruit participants for this study. Following the observations and interviews, we used the grounded theory approach to analyze the qualitative data [1]. An affinity diagram was built on our project room walls and insights were developed from a clustering of collected data.

\begin{tabular}{|l|l|}
\hline \multicolumn{2}{|c|}{ Chicagoland area (U.S.) } \\
\hline Downtown Loop & Lincoln Square \\
\hline Wicker Park & Pilsen \\
\hline Logan Square & Hyde Park \\
\hline River North & Evanston \\
\hline Little Italy & Naperville \\
\hline Streeterville & Oak Lawn \\
\hline
\end{tabular}

Figure 1: Geographic areas covered in research

After conducting interviews with people from all over Chicago, our biggest insight was that there are six high-level criteria that comprise the decisionmaking process for trip planning: time/duration, convenience, cost, comfort, safety, and health/fitness.

The most frequently narrated compromise made by people was between using Uber/Lyft and riding the public bus/subway. Uber/Lyft is extremely convenient but quite expensive compared to public transportation. The public bus/subway, on the other hand, is very cost-effective, yet is inconvenient because of its fixed pickup/drop-off locations and overcrowding during peak travel hours. Current transportation systems optimize for these two criteria the most and so as a consequence people mention them the most. The criteria of comfort, safety and health/fitness are also optimized but at a personal level and more implicitly by the user.

We re-evaluated current offerings (apps and maps) with these six criteria. We discovered, for instance, that the Google Maps application only takes into account time/duration, in effect assuming that's the only criterion users consider. Citymapper goes a bit further and displays estimated calories a traveler would burn if they decide to walk or ride a bicycle, thus allowing users to compare both the time/duration and health/fitness benefits of a route. This is certainly a step up, but still leaves many other considerations unaccounted for, such as safety.

Furthermore, current offerings often display unnecessary information, adding irrelevant complexity to the interface for trip planning. Both Google Maps and Citymapper suggest routes in multiple modes of transport, many of which are irrelevant to users. Additionally, Google Maps has the practice of displaying two alternative routes to each destination, yet not providing any explicit advantage, as the routes are often labeled with only a "Similar ETA." These two examples illustrate the unnecessary complexity in current interfaces and the overall user experience of current offerings.

For concept development, we walked the wall of post-its and brainstormed ideas that addressed the user needs. Smaller ideas were merged and a full solution was developed. For early user evaluation and feedback, a low-fidelity interactive prototype was developed in the Principle for Mac software and demonstrated on actual phones to 10 different individuals (ages 25-65). We video recorded the interactions of each individual and asked them to think aloud as they went through the setup phase and onto exploring the features of Sway.

\section{CONCEPT DEVELOPMENT}

Based on our findings, we set out to create a mobile platform that could account for all six criteria and provide travelers with a unique travel experience. Our goal was to design an interface that would only display modes, information, and routes that a user would find helpful and hide everything else that's irrelevant to the specific context. To achieve this, we created a system called "Sway" that would learn a user's preferences upon repeated usage and recommend routes accordingly.

\subsection{How Sway Works}

Sway is built around a structure we call a "smart scaffolding" or "Tier 1." This structure displays three trip options, based on a handful of setup questions aimed at understanding the user's preferences at a 


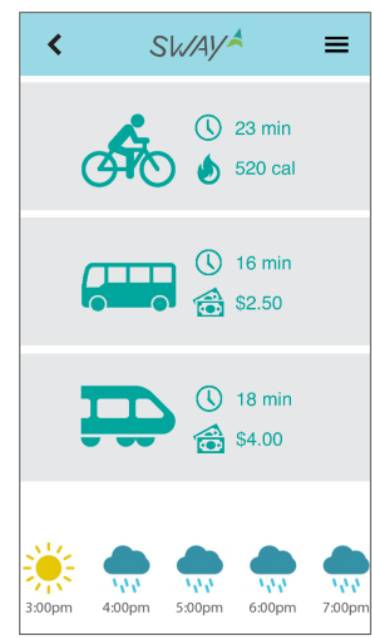

Figure 2: Tier 1 optimization

basic level. Each option would feature a mode of transport or combination of modes that prioritizes one of the six criteria. For example, based on one of the personas we developed from our research, the first option may be to ride a bicycle because Sway has learned that health/fitness is important to the user. The second option could be to take the bus because Sway has learned that costeffectiveness is also a priority to the user.

In addition to a mode of transportation, each option would display relevant information, such as duration of the trip, cost of the trip, and calories burned. The display of these types of information would prove particularly effective for users to whom cost-effectiveness and health/fitness are important (see Figure 2). When the user selects an option, their decision is logged, reinforcing the criteria connected to that option while simultaneously deemphasizing other criteria that was ignored by the user.

After selecting one of the three trip options, a user is brought to "Tier 2," which would display a map view with at least two routes. As with the previous Tier, each route would be connected to a different criterion. For example, one route would be "Shortest time," which would take the time/duration criterion as its priority. Another route would be "Safest," which would take the safety criterion as its priority (see Figure 3). "Safety" when it comes to biking, would be determined/calculated on the availability of clearly marked bike lanes as well as recorded city data of bike accident incidents. Users could easily toggle between routes and the information at the top would adjust accordingly as well as have a more distinctive route variability/tagging then from what is currently being offered by other apps (see Figure 4).

To further aid Sway's accuracy in learning a user's travel preferences, Sway would tag every user-

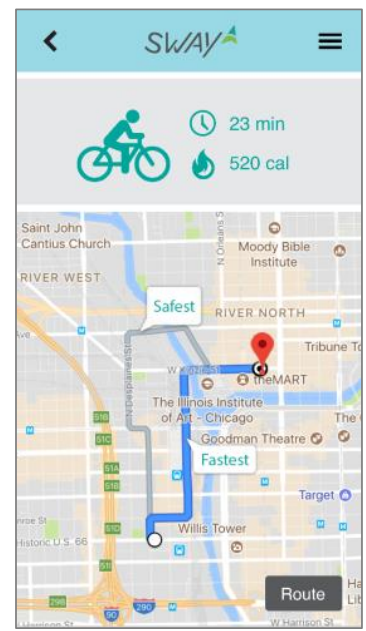

Figure 3: Tier 2 optimization

selected option with a set of information to record the circumstances in which that option was selected. This would allow Sway to understand the context in which the user chose a particular option or route. Sway would tag the 1) day of the week, 2) time of day, 3) date, 4) weather, 5) nearby traffic patterns, 6) nearby special events (e.g. concerts, sporting events), 7) events in the user's calendar around the time of the selection, and 8) transportation delays related to the selection. In the future under similar circumstances, Sway would have a point of reference.

\begin{tabular}{|l|l|}
\hline Tier $\mathbf{1}$ & Tier $\mathbf{2}$ \\
\hline Mode of transport & Fastest route \\
\hline Time/duration & Safest route \\
\hline Cost & Least transfers \\
\hline Calories burned & Least traffic \\
\hline \multirow{2}{*}{} & Shortest distance \\
\cline { 2 - 2 } & $\begin{array}{l}\text { Most scenic route/ } \\
\text { Tourist mode }\end{array}$ \\
\cline { 2 - 2 }
\end{tabular}

Figure 4: Type of relevant information Sway shows in each Tier to aid user's decision-making

\subsection{User Evaluation of Concept}

Although limited in its functionality, the low-fidelity prototype gave people a relatively high-resolution user experience of the concept. People validated several needs and questioned certain features.

Our prototype was optimized for a person who values health/fitness, so biking was the first recommended option in Tier 1 . From there, the user would be presented with two routes in Tier 2: 1) the fastest route and 2) the safest route. 
Other features that users explored included a message center, a weather scroll, a dashboard to view travel expenses with budget goal setting, and a dashboard to view health/fitness activity with related goal setting. Users were also presented with the possibility of connecting to other existing platforms such as Mint, Fitbit, and Google in order to drive/provide better aggregate data and suggestions.

\subsection{User Evaluation Findings}

Our testers responded positively to our "smart scaffolding" in Tier 1, commenting that simplifying trip planning to three recommended options made the process easier. Tier 2 received an even greater positive response as people loved having multiple yet distinctive, highly personalized routing options as a feature.

Two major insights emerged from our testing. The first was that users have different considerations for different modes of transportation. Knowing the safest route may be important to a user when riding a bicycle, but not when they're driving. The second was that while a user's overarching travel preferences are consistent, there may be exceptions in certain situations. For example, suppose a user unexpectedly finds themselves with extra time to drive to their destination. Perhaps this one time they'd prefer to take the route with least traffic instead of the route with the shortest travel time. We found that making adjustments to the routes in Tier 2 is an effective way to incorporate both of these insights.

As for other features, we were surprised that the ability to optimize for a given budget received negative feedback. It was seen as a good idea but many users were hesitant to connect their finances with a new, unproven app. One user suggested showing comparison spending or 'what might've been' if a user where to take other modes of transportation. The reception for the health/fitness feature was relatively neutral, but we did note a handful of people referring to other fitness applications they use. The ability to integrate data from those apps is a possible area for exploration.

\section{DISCUSSION}

\subsection{Just the right amount of complexity needs to be shown}

Each person has a unique set of preferences that change dynamically from one circumstance to another. We observe that oversimplification of user interface doesn't address the needs of users neither does it enhance the usability of the user interface. The only way to simplify the experience is to personalize the options/routes and show only the ones that are relevant - which could be more than one due to personal preferences as well as situational needs. The options/routes need to be significantly different from one another to enable an easier informed decision. Sway's smart scaffolding provides a way for exposing a certain level of complexity to our users while simultaneously maintaining the trip planning experience quite personal and simple.

\subsection{Lower barriers to unfamiliar modes and routes and enable discovery}

Sway makes it easier for users to discover new modes of transportation and routes that better fulfil their needs. As a result, when the user sees an option they're unfamiliar with yet fitting their needs, there is better chance that they might try it. This could have large implications on travel behavior, as travelers' trips become increasingly efficient while allowing for the spirit of serendipity and adventure.

\subsection{Sway adapts in real time to user needs and behaviors making it situationally aware}

Sway is constantly learning and adjusting to a user's preferences, which will help users build trust in the system's recommendations. With overwhelming choice in transportation it is imperative that Sway learn over time about the travel habits of users as well their situational optimizations. When deployed for making user choices more informed, Sway will be able to sway people with a timely nudge to make travel choices that are sustainable, healthy and more importantly fun in mundane circumstances of everyday life.

\section{NEXT STEPS}

We are looking to create a second prototype for user testing of concept(s) and eventually we plan to build a hi-fidelity functional prototype for in-field trials in the city of Chicago.

\section{REFERENCES}

1. Hugh Beyer, and Karen Holtzblatt. 1998. Contextual Design: Defining Customer-Centered Systems. Morgan Kaufmann Publishers.

\section{Citymapper.com}

3. Julian Dibbelt et al., "Multimodal route and tour planning in urban environments," 2017 IEEE Symposium on Computers and Communications (ISCC), Heraklion, 2017, pp. 214-219.

4. Claas Digmayer, Sara Vogelsang, and EvaMaria Jakobs. 2015. Designing mobility apps to support intermodal travel chains. In Proceedings 
of SIGDOC 2015. ACM, New York, NY, USA, Article 44, 11 pages.

5. Eric Goldwyn. 2014. The Most Important Transportation Innovation of the Decade is the Smartphone. Retrieved January 4, 2018 from https://www.citylab.com/transportation/2014/09/t he-most-important-transportation-innovationofthis- decade-is-the-smartphone/379525/

6. Hanna Hasselqvist, Mia Hesselgren, and Cristian Bogdan. 2016. Challenging the Car Norm: Opportunities for ICT to Support Sustainable Transportation Practices. In Proceedings of the $2016 \mathrm{CHI}$ Conference on Human Factors in Computing Systems (CHI '16), 1300-1311.

7. Laura Magrini, Matteo Nati, and Emanuele Panizzi. 2012. RMob - a mobile app for real time information in urban transportation. In Proceedings of AVI 2012, Genny Tortora, Stefano Levialdi, and Maurizio Tucci (Eds.). ACM, New York, NY, USA, 776-777.

8. Maps.google.com

9. Susan Shaheen, and Matt Christensen. 2014. The True Future of Transportation Has Two Big Barriers. Retrieved January 4, 2018 from https://www.citylab.com/transportation/2014/04/tr ue-future-transportation-has-two-bigbarriersentry/ 8933/

10. Caleb Southern, Yunnuo Cheng, Cheng Zhang, and Gregory Abowd. 2017. Understanding the Cost of Driving Trips. In Proceedings of the 2017 $\mathrm{CHI}$ Conference on Human Factors in Computing Systems (CHI '17). ACM, NY, USA, 430-434.

11. Martin Stein, Johanna Meurer, Alexander Boden, and Volker Wulf. 2017. Mobility in Later Life: Appropriation of an Integrated Transportation Platform. In Proceedings of the 2017 CHI Conference on Human Factors in Computing Systems (CHI '17). ACM, New York, NY, USA, 5716-5729.

12. Harold Thimbleby, Ann Blandford, Paul Cairns, Paul Curzon, and Matt Jones. 2012. User Systems Design as Systems Design. Faulkner $X$., Finlay J., Détienne F. (eds) People and Computers XVI - Memorable Yet Invisible 